\title{
РАЗРАБОТКА SVAR МОДЕЛИ ДЛЯ ЦЕЛЕЙ ОПРЕДЕЛЕНИЯ РЕАКЦИИ АКТИВОВ НА СЧЕТАХ ПРОФЕССИОНАЛЬНЫХ УЧАСТНИКОВ РЫНКА ЦЕННЫХ БУМАГ НА СТРУКТУРНЫЕ ШОКИ
}

\author{
(C) 2021 Гребенькова Мария Алексеевна \\ магистрант \\ Финансовый университет при Правительстве Российской Федерации, Россия, Москва \\ E-mail: mariya_grebenkova@mail.ru \\ (c) 2021 Толмачев Михаил Николаевич \\ доктор экономических наук, доцент, профессор, департамент бизнес-аналитики \\ Финансовый университет при Правительстве Российской Федерации, Россия, Москва \\ E-mail: MNTolmachev@fa.ru
}

В работе предлагается модель структурной векторной авторегрессии для определения реакций объемов активов клиентов профессиональных участников рынка ценных бумаг на монетарный структурный шок. Разработка данной модели с учетом специфических для рассматриваемых профессиональных участников (брокеры и управляющие компании) переменных является целью данного исследования. Актуальность исследования обусловлена активным внедрением практики стресс-тестирования профессиональных участников, так как выявленные реакции переменных, отвечающих за динамику активов клиентов профессиональных участников, на структурные шоки могут быть использованы в гипотетических стрессовых сценариях. Идентификация структурных шоков в рамках предложенных моделей осуществлена с использованием подхода разложения ковариационной матрицы остатков по Холецкому. Полученные результаты согласуются с проведенным на предварительном этапе анализом рассматриваемых временных рядов и принятых на его основании экономических предпосылок. Расчеты использованы с использованием программной среды R Studio. Полученные результаты могут быть использованы для целей макроэкономического прогнозирования объемов изъятия активов пайщиками и клиентами брокерских компаний, а также для целей стресс-тестирования профессиональных участников рынка ценных бумаг.

Ключевые слова: модель структурной векторной авторегрессии, профессиональные участники рынка ценных бумаг, функции импульсного отклика, структурные шоки, стресс-тестирование

\section{Введение}

Финансовая устойчивость и платежеспособность финансовых посредников подвержена существенному влиянию риска оттока активов клиентов. Но, если вопросу оттока депозитов клиентов кредитных организаций посвящен широкий пласт исследований, вопрос изъятия активов клиентами профессиональных участников рынка ценных бумаг (ПУ РЦБ) изучен не в полной мере. При этом риску изъятия активов отведена лидирующая позиция в списке рисков профессиональных участников, определенных Европейским управлением по надзору за рынком ценных бумаг (ESMA), для целей стресстестирования", так как он способен привести к существенному ухудшению финансового поло- жения ПУ РЦБ в результате принудительного закрытия позиций в активах, резкого сокращения объема активов в части денежных средств. Целью исследования является разработка модели структурной векторной авторегрессии (SVAR), позволяющей определять уровень оттока активов клиентов профессиональных участников в условиях структурных шоков.

\section{Спецификация модели}

Структурная векторная авторегрессия относится к классу моделей векторной авторегрессии, получившими распространение после публикации К.Симса [7]. Преимуществами всего класса векторных авторегрессий являются отсутствие необходимости разделения переменных на экзогенные и эндогенные и устранение

\footnotetext{
* ESMA Economic Report Stress simulation for investment funds. European Securities and Markets Authority, Paris. 2019. URL: https://www.esma.europa.eu/sites/default/files/library/esma50-164-2458_stresi_report.pdf (Дата обращения 13.05.2021).
} 
сложностей, присущих системам одновременных уравнений.

В общем виде модель структурной векторной авторегрессии записывается как следующее уравнение:

$\mathrm{B}_{0} \mathrm{Y}_{\mathrm{t}}=\mathrm{B}_{1} \mathrm{Y}_{\mathrm{t}-1}+\ldots+\mathrm{B}_{\mathrm{p}} \mathrm{Y}_{\mathrm{t}-\mathrm{p}}+\overrightarrow{\mathrm{w}_{\mathrm{t}}}$

где $\mathrm{Y}_{\mathrm{t}}$ - вектор эндогенных переменных $(\mathrm{n} \times 1)$;

$\mathrm{B}_{0}$ - матрица неидентифицируемых параметров;

$\overrightarrow{\mathrm{w}_{\mathrm{t}}}$ - вектор структурных шоков, являющихся взаимно некоррелированным белым шумом с диагональной ковариационной матрицей $\sum \mathrm{w}$, один или более из элементов $\overrightarrow{\mathrm{w}_{\mathrm{t}}}$ имеют определенную экономическую интерпретацию.

Структурные шоки, как правило, не наблюдаемы и не обязательно должны быть связаны с какой-либо переменной из соответствующей VAR модели.

Выделяют следующие подходы к идентификации шоков в модели SVAR [1]:

1) к оцененной матрице ковариации остатков (ошибок) применяется разложение Холецкого, что влечет строгое предположение об экономической структуре;

2) формулировка структурных уравнений для ошибок, например, таких как уравнение кривой инвестиции - сбережения, кривой спроса на деньги;

3) ограничение на долгосрочный эффект шоков (в том числе знаковые ограничения).

Типичными идентифицируемыми в рамках SVAR шоками являются шок совокупного спроса, совокупного предложения, монетарный, фискальный, технологический шок [3]. Л. Килиан и Ч. Парк [4] в целях выявления влияния структурных изменений на рынке нефти на доходность фондового рынка включают в модель SVAR три вида структурных шоков, свойственные рынку нефти (шок совокупного спроса, шок спроса на нефть, шок предложения нефти) и один шок, не обязательно структурный, влияющий на доходность фондового рынка США. Л.Гамбеттии А. Муссо [2] развивают идею влияния шока предложения банковских ссуд на экономическую активность (прирост ВВП), инфляцию (прирост цен на потребительские товары) и кредитные условия (краткосрочная процентная ставка) ме- тодами пятифакторной (пятым фактором является вектор неучтенных шоков) структурной векторной авторегрессионной модели с изменяющимися во времени параметрами (TVP SVAR). В работах $[5,4,2]$, развитых в рамках стандартной структурной векторной авторегрессии, число эндогенных переменных, функции отклика которых требуется изучить, соответствует числу шоков (экзогенных процессов), влияние которых оценивается в модели.

Структурный (экзогенный) шок понимается нами как неожиданное изменение в определенной эндогенной переменной, отвечающей за макроэкономическую конъюнктуру (уровень реального ВВП, уровень инфляции) либо за специфические для профессиональных участников условия (объем активов клиентов на брокерском счете, активов под управлением, клиентского торгового оборота).

В работе рассматривается два вида финансовых посредников из числа ПУ РЦБ: брокерские компании и управляющие компании. Дилеры не принимаются во внимание, так как данная бизнес-модель не предполагает существенной доли клиентских активов в структуре баланса, а также, потому что зачастую бизнес-модели брокера и дилера объединены в деятельности одного профессионального участника.

Поведение клиентской базы брокеров может быть описано объемом клиентских операций, так как торговый оборот влияет на уровень получаемого брокерами комиссионного дохода, в то время как поведение клиентской базы управляющих компаний (пайщиков) стоит описывать динамикой стоимости чистых активов, очищенной от доходности. Выбор переменных был обусловлен наличием доступной информации. Так, в качестве переменной, отвечающей за поведение пайщиков, были использованы квартальные данные по объему финансовых активов под управлением инвестиционных фондов, публикуемый OECD* (aum), а в качестве переменной, отвечающей за поведение клиентской базы брокерских компаний - квартальные данные по суммарному стоимостному объему сделок, заключенных в интересах клиентов 10 ведущих операторов (deals), рассчитанные как простое среднее по месячным данным, публикуемым Московской биржей**. Помимо анализа

\footnotetext{
* Официальный сайт The Organisation for Economic Co-operation and Development (OECD). URL: https://stats. oecd.org/Index.aspx? DataSetCode=QASA_7II\# (Дата обращения: 13.05.2021).

** Официальный сайт Московской биржи. URL: https://www.moex.com/s719 (Дата обращения: 13.05.2021).
} 
торгового оборота, внимания заслуживает анализ динамики числа активных клиентов (client): высокая концентрация активов на счетах брокерского обслуживания может быть причиной резкого неожиданного сокращения активов в отсутствии способствующих тому макроэкономических предпосылок, так как, согласно данным Банка России, на 5,7\% счетов сконцентрировано 93,9\% активов розничных инвесторов, а на $1 \%$ крупнейших счетов - $64 \%$ клиентских активов*.

В качестве переменных макроэкономической конъюнктуры использованы квартальные данные по реальному ВВП в ценах 2016 года, сезонно скорректированные (real_gdp_sa), уровню инфляции (срі), ставке денежного рынка MIACR (miacr), значению фондового индекса Мосбиржи (imoex), курсу национальной валюты по отношению к долл. США (ex_rate) Анализ покрывает период с III кв. 2007 г. по III кв. 2020 г., так что исходный ряд по каждой переменной состоит из 56 наблюдений.

Важным условием проведения анализа временных рядов методом SVAR является стационарность рассматриваемых рядов, поэтому все переменные были подвергнуты определенным модификациям: 1) логарифмирование $\left(\ln z_{t}\right)$; 2) расчет прироста логарифма $\left(\ln z_{t}-\ln z_{t-1}\right)$. Результаты данных модификаций и значения тестовых статистик расширенного теста ДикаФуллера на единичность корня (ADF-тест) представлены в таблице 1.

В качестве нулевой гипотезы в рамках теста Дика-Фуллера [6] принимается предположение о наличии единичного корня. В результате данного тестирования было выявлено, что в отношении всех рассматриваемых временных рядов за исключением ряда количества активных клиентов (client) имеет место интегрированность первого порядка, так как логарифмы данных рядов нестационарны, в то время как стационарны их первые разности. Из таблицы 3 следует, что количество активных клиентов является нестационарным рядом даже после преобразования этого ряда в ряд логарифмических приростов, что объясняет исключение данной переменной из дальнейшего анализа. Графики отобранных переменных в преобразованном виде представлены на Рисунке 1.

Из рисунка 1 следует, что динамика логарифмических приростов объема финансовых активов под управлением (diff_ln_aum) во многом

* Официальный сайт Банка России. URL: https://cbr.ru/analytics/rcb/broker_market/ (Дата обращения: 13.05.2021).

Таблица 1. Результаты расширенного теста Дика-Фуллера на единичность корня

\begin{tabular}{|c|c|c|c|c|c|c|c|}
\hline \multirow{2}{*}{ Переменная } & \multirow{2}{*}{ Модель теста } & \multirow{2}{*}{ Лаг } & \multirow{2}{*}{$\begin{array}{c}\text { Тестовая } \\
\text { статисти- } \\
\text { ка }\end{array}$} & \multicolumn{3}{|c|}{ Критические значения } & \multirow{2}{*}{ p-value } \\
\hline & & & & $1 \%$ & $5 \%$ & $10 \%$ & \\
\hline \multicolumn{8}{|c|}{ Макроэкономические переменные } \\
\hline ln_real_gdp_sa & constant, trend & 2 & $-3,739$ & $-4,04$ & $-3,45$ & $-3,15$ & 0,0299 \\
\hline diff_ln_real_gdp_sa & none & 1 & $-4,06$ & $-2,6$ & $-1,95$ & $-1,61$ & $<0,01$ \\
\hline ln_cpi & constant, trend & 1 & $-5,3197$ & $-4,04$ & $-3,45$ & $-3,15$ & $<0,01$ \\
\hline diff_ln_cpi & none & 1 & $-9,5796$ & $-2,6$ & $-1,95$ & $-1,61$ & $<0,01$ \\
\hline miacr & constant, trend & 2 & $-1,6738$ & $-4,04$ & $-3,45$ & $-3,15$ & 0.7058 \\
\hline diff_miacr & none & 1 & $-4,9611$ & $-2,6$ & $-1,95$ & $-1,61$ & $<0,01$ \\
\hline ln_imoex & none & 1 & 0,2764 & $-2,6$ & $-1,95$ & $-1,61$ & 0,699 \\
\hline diff_ln_imoex & none & 1 & $-5,4842$ & $-2,6$ & $-1,95$ & $-1,61$ & $<0,01$ \\
\hline ln_ex_rate & constant, trend & 1 & $-2,2508$ & $-4,04$ & $-3,45$ & $-3,15$ & 0,4735 \\
\hline diff_ln_ex_rate & none & 1 & $-5,0129$ & $-2,6$ & $-1,95$ & $-1,61$ & $<0,01$ \\
\hline \multicolumn{8}{|c|}{ Специфические для ПУ РЦБ переменные } \\
\hline ln_aum & constant, trend & 2 & $-2,7933$ & $-4,04$ & $-3,45$ & $-3,15$ & 0,2551 \\
\hline diff_ln_aum & none & 1 & $-4,1143$ & $-2,6$ & $-1,95$ & $-1,61$ & $<0,01$ \\
\hline ln_deals & constant, trend & 3 & $-2,7718$ & $-4,04$ & $-3,45$ & $-3,15$ & 0,2638 \\
\hline diff_ln_deals & none & 2 & $-3,9723$ & $-2,6$ & $-1,95$ & $-1,61$ & $<0,01$ \\
\hline ln_client & none & 3 & 1,131 & $-2,6$ & $-1,95$ & $-1,61$ & 0,9277 \\
\hline diff_ln_client_sa & constant & 1 & $-1,3529$ & & & & 0,5535 \\
\hline
\end{tabular}




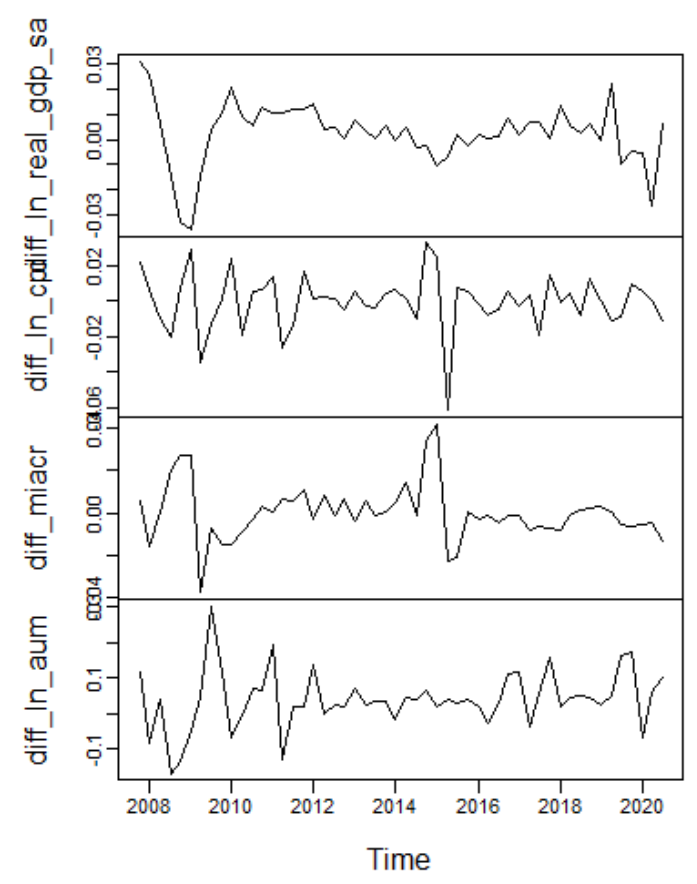

data_fd

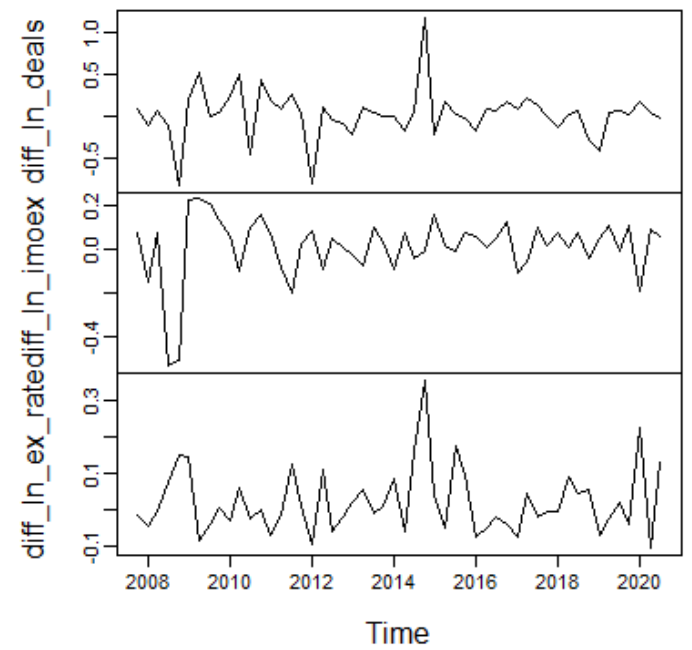

Рисунок 1. Динамика переменных, используемых в модели *, III кв. 2007 - III кв. 2020 гг. * А именно: реальный ВВП, сезонно скорректированный; объем финансовых активов под управлением, клиентский торговый оборот, ИПЦ, ставка денежного рынка, фондовый индекс, курс рубля к долл. США.

повторяет тренд логарифмических приростов реального ВВП (сезонно скорректированного) (diff_ln_real_gdp_sa), в то же время объем торгового оборота клиентов ведущих операторов биржи (diff_ln_deals) не может быть объяснен динамикой реального ВВП, но резкий прирост торгового оборота в 2015 году совпадает с резким приростом ставки денежного рынка (diff_miacr) и падением курса рубля по отношению к долл. США (diff_ln_ex_rate) в этот период (валютный кризис). Также стоит отметить одновременный резкий спад активов под управлением и клиентского торгового оборота, приходящийся на 2009 год, и сопутствующая им негативная динамика фондового индекса (diff_ln_imoex).

Проведенный тест Йохансена на коинтеграцию обнаружил наличие общих детерминированных трендов в уравнениях регрессии, связывающих: 1) ставку денежного рынка и фондовый индекс; 2) курс рубля к доллару и фондовый индекс; 3) фондовым индекс и объем денежных средств под управлением, 4) фондовый индекс и клиентский торговый оборот (результаты представлены в таблице 2). Данные результаты могут быть использованы в дальнейшем для целей спецификации модели.

Таблица 2. Результаты теста Йохансена

\begin{tabular}{|c|c|c|c|c|}
\hline Комбинация рядов & $\mathrm{R}<=$ & $\begin{array}{c}\text { Нет тренда, нет кон- } \\
\text { станты }\end{array}$ & $\begin{array}{c}\text { Нет тренда, есть кон- } \\
\text { станта }\end{array}$ & $\begin{array}{c}\text { Есть тренд, есть кон- } \\
\text { станта }\end{array}$ \\
\hline MIACR - $\ln (\mathrm{IMOEX})$ & 0 & 8,68 (незначим) & 8,84 (незначим) & $\begin{array}{l}27,91 \text { (значим на } 5 \% \\
\text { уровне) }\end{array}$ \\
\hline $\ln (\mathrm{IMOEX})-\ln (\mathrm{AUM})$ & 0 & $\begin{array}{l}21,05 \text { (значим на } 5 \% \\
\text { уровне) }\end{array}$ & $\begin{array}{l}35,44 \text { (значим на } 1 \% \\
\text { уровне) }\end{array}$ & $\begin{array}{l}28,44 \text { (значим на } 5 \% \\
\text { уровне) }\end{array}$ \\
\hline $\ln (\mathrm{IMOEX})-\ln (\mathrm{DEALS})$ & 0 & 15,34 (незначим) & 15,96 (незначим) & $\begin{array}{l}27,39 \text { (значим на } 5 \% \\
\text { уровне) }\end{array}$ \\
\hline $\ln (\mathrm{EX}-\mathrm{RATE})-\ln (\mathrm{IMOEX})$ & 0 & $\begin{array}{l}16,77 \text { (значим на } 10 \% \\
\text { уровне) }\end{array}$ & $\begin{array}{l}21,38 \text { (значим на } 5 \% \\
\text { уровне) }\end{array}$ & $\begin{array}{l}27,02 \text { (значим на } 5 \% \\
\text { уровне) }\end{array}$ \\
\hline $\ln (\mathrm{EX}-\mathrm{RATE})-\ln (\mathrm{AUM})$ & 0 & 8,49 (незначим) & $\begin{array}{l}20,93 \text { (значим на } 5 \% \\
\text { уровне) }\end{array}$ & 22,09 (незначим) \\
\hline $\ln ($ EX-RATE $)-\ln ($ DEALS $)$ & 0 & 8,31 (незначим) & 11,78 (незначим) & 13,68 (незначим) \\
\hline
\end{tabular}


Рассмотрим модель структурной векторной авторегрессии в условиях следующих структурных шоков:

1) монетарный шок, влекущий повышение ставки денежно-кредитной политики (ключевой ставки), аппроксимированной ставкой денежного рынка MIACR;

2) монетарный шок, влекущий ослабление курса национальной валюты по отношению к долл. США.

Проведенный на предварительном этапе анализ дает основания для введения определенных предпосылок о характере связей между рассматриваемыми эндогенными переменными:

1) структурный шок, влияющий на динамику курса рубля по отношению к долл. США, влияет также на фондовый индекс (поскольку обнаружен общий детерминированный тренд);

2) структурный шок, влияющий на ставку денежного рынка, оказывает влияние на фондовый индекс (общий детерминированный тренд);

3) динамика фондового индекса влияет на динамику активов профессиональных участников.

С учетом принятых допущений задается следующий вектор эндогенных переменных для случая монетарного шока, являющегося причиной повышения ставки MIACR:

$$
\mathrm{Y}_{1}=\left(\begin{array}{c}
\Delta \text { CPI } \\
\Delta \text { GDP } \\
\Delta \text { MIACR } \\
\Delta \text { IMOEX } \\
\Delta \text { AUM } / \Delta \text { DEALS }
\end{array}\right)
$$

Для случая монетарного шока, влекущего ослабление курса рубля к долл. США, вектор эндогенных переменных принимает вид:

$$
\mathrm{Y}_{2}=\left(\begin{array}{c}
\Delta \text { CPI } \\
\Delta \text { GDP } \\
\Delta \text { MIACR } \\
\Delta \text { RUB } / \text { USD } \\
\Delta \text { AUM } / \Delta \text { DEALS }
\end{array}\right)
$$

В работе рассматривается две бизнесмодели профессиональной деятельности на рынке ценных бумаг: деятельность по управлению ценными бумагами (УК) и брокерская деятельность, в связи с этим каждая модель рассчитывается по-отдельности для бизнес-модели УК и для бизнес-модели брокера. Поэтому «наиболее эндогенная» переменная для первой бизнесмодели - активы под управлением (AUM), а для второй - клиентский торговый оборот (DEALS).

Идентификация структурных шоков в рассматриваемых моделях задается краткосрочными ограничениями: во всех сценариях применяется подход, основанный на разложении ковариационной матрицы остатков по Холецкому, что требует упорядочения переменных, таким образом, чтобы «наиболее неэндогенная» переменная из рассматриваемых стояла на первом месте, а «наиболее эндогенная» (то есть та, на которую оказывают влияние все шоки) - на последнем.

Матрица ограничений в рамках подхода разложения Холецкого для случая монетарного шока в результате повышения ставки задается следующим уравнением:

$$
\left(\begin{array}{c}
u^{\Delta \text { CPI }} \\
u^{\Delta \text { GDP }} \\
u^{\Delta \text { MIACR }} \\
u^{\Delta \text { IMOEX }} \\
u^{\Delta \text { AUM } / \Delta \text { DEALS }}
\end{array}\right)=\left[\begin{array}{ccccc}
b_{0}^{11} & 0 & 0 & 0 & 0 \\
b_{0}^{21} & b_{0}^{22} & 0 & 0 & 0 \\
b_{0}^{31} & b_{0}^{32} & b_{0}^{33} & 0 & 0 \\
b_{0}^{41} & b_{0}^{42} & b_{0}^{43} & b_{0}^{44} & 0 \\
b_{0}^{51} & b_{0}^{52} & b_{0}^{53} & b_{0}^{54} & b_{0}^{55}
\end{array}\right] \times\left(\begin{array}{c}
w_{1 t} \\
w_{2 t} \\
w_{3 t} \\
w_{4 t} \\
w_{5 t}
\end{array}\right)
$$

Структурный шок воздействует на каждую из эндогенных переменных, он является причиной изменения уровня цен и ВВП, поэтому может быть интерпретирован как смещение кривой совокупного предложения. Шок является причиной изменения динамики ВВП, но не влияет на уровень цен, поэтому он будет отражать смещение кривой совокупного спроса. Шок влияет на ставку денежного рынка, динамику фондового индекса и объем активов на счетах профессиональных участников, и может быть реакцией денежно-кредитных властей на объясненные выше шоки совокупного предложения и совокупного спроса, поэтому он интерпретируется как шок монетарной политики. Шок может быть представлен в качестве специфического для фондового рынка шока спроса на финансовые инструменты. Шок вбирает в себя все оставшиеся неучтенные шоки и является не обязательно структурным шоком.

Аналогичным образом специфицируется модель, в которой монетарный шок влияет на динамику курса национальной валюты к долл. США:

$$
\left(\begin{array}{c}
u^{\Delta C P I} \\
u^{\Delta G D P} \\
u^{\Delta U S D / R U B} \\
u^{\Delta I M O E X} \\
u^{\Delta \text { AUM } / \triangle \text { DEALS }}
\end{array}\right)=\left[\begin{array}{ccccc}
b_{0}^{11} & 0 & 0 & 0 & 0 \\
b_{0}^{21} & b_{0}^{22} & 0 & 0 & 0 \\
b_{0}^{31} & b_{0}^{32} & b_{0}^{33} & 0 & 0 \\
b_{0}^{41} & b_{0}^{42} & b_{0}^{43} & b_{0}^{44} & 0 \\
b_{0}^{51} & b_{0}^{52} & b_{0}^{53} & b_{0}^{54} & b_{0}^{55}
\end{array}\right] \times\left(\begin{array}{c}
w_{1 t} \\
w_{2 t} \\
w_{3 t} \\
w_{4 t} \\
w_{5 t}
\end{array}\right)
$$


Только в этой спецификации монетарный шок влияет на обменный курс, а не на ставку денежного рынка, соответственно динамика фондового индекса и объемов активов на счетах профессиональных участников находятся под воздействием динамики обменного курса.

\section{Результаты}

Для целей определения воздействия монетарных шоков на объем активов ПУ РЦБ были определены функции импульсных откликов в рамках каждой из рассматриваемых моделей. В свою очередь, для рассматриваемых SVAR моделей были оценены параметры соответствующий VAR моделей. Результаты проверки рассматриваемых моделей векторной авторегрессии подтвердили состоятельность полученных оценок.

В отношении сценария монетарного шока, влияющего на повышение ставки денежного рынка, было выявлено, что в случае единичного изменения данной с учетом заданных ограничений для возвращения на равновесную траекторию объема активов под управлением и объ- ема клиентского торгового оборота потребуется 10 кварталов (рис. 2). В течение года ожидается логарифмический прирост в 0,01 п.п. объема активов под управлением (бизнес-модель УK), в 0,025 п.п. клиентского торгового оборота (бизнес-модель брокера) после спада до $-0,05$ п.п. Функции импульсных откликов объемов активов под управлением и клиентского торгового оборота на единичный шок ставки денежного рынка изображены сплошной линией, границы 95\%-х доверительных интервалов для данных реакций - пунктирными линиями.

Реализация модели SVAR в рамках сценария монетарного шока, влияющего на курс рубля к долл. США, показала, что объем активов под управлением в случае единичного прироста значения USD/RUB (что означает ослабление курса рубля), в логарифмических приростах увеличивается более чем на 0,01 п.п., в то время как объем клиентского торгового оборота сокращается на 0,1 п.п. в течение года (рис. 3).
SVAR Impulse Response from diff_miacr

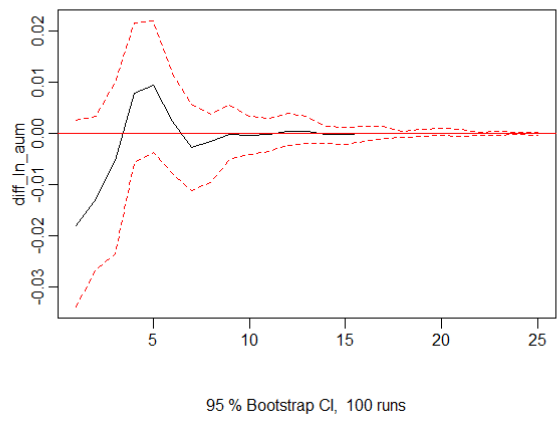

а) бизнес-модель УК
SVAR Impulse Response from diff_miac

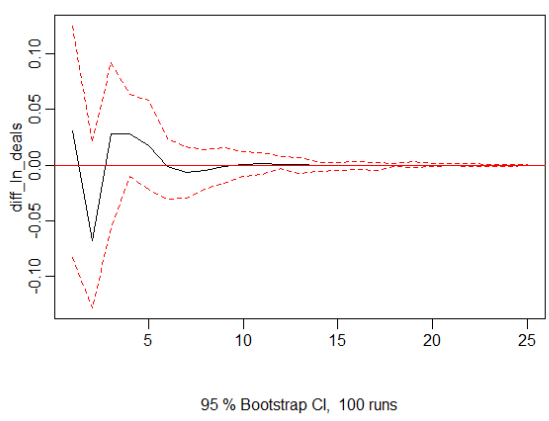

б) бизнес-модель брокера

Рисунок 2. Функции импульсного отклика переменных объема активов под управлением (diff_ln_aum) и клиентского торгового оборота (diff_ln_deals) при единичном изменении MIACR

SVAR Impulse Response from diff_In_ex_rate

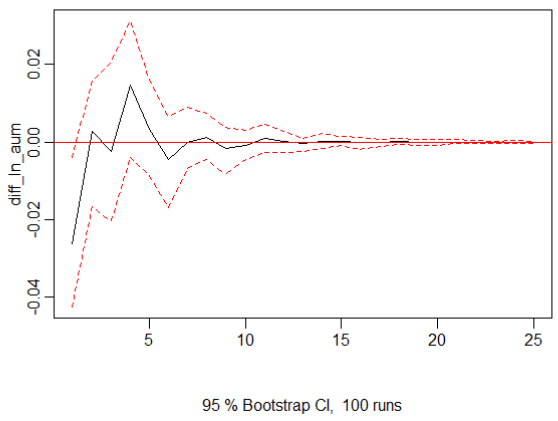

а) бизнес-модель УК

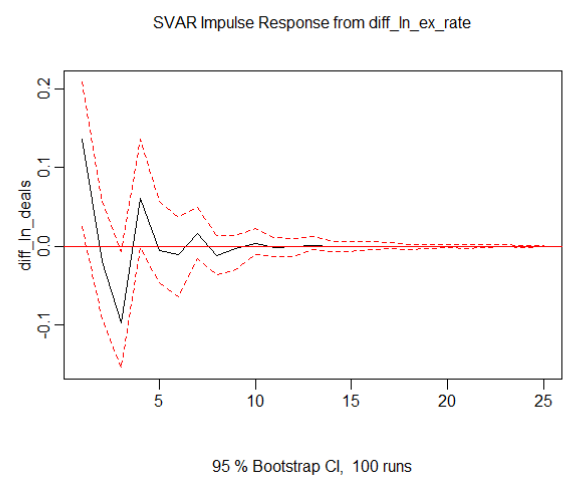

б) бизнес-модель брокера

Рисунок 3. Функции импульсного отклика переменных объема активов под управлением (diff_ln_aum) и клиентского торгового оборота (diff_ln_deals) на единичное изменение USD/RUB 
В результате проведенного исследования было выявлено, что объем активов под управлением и клиентский торговый оборот разнонаправленно реагируют на монетарные шоки.

В целях дальнейшего развития анализа влияния структурных шоков на специфические для профессиональных участников параметры, закладываемые в модели стресс-тестирования, предполагается, что перечень рассматриваемых в работе переменных может быть расширен. В связи с этим оправданным будет использование инструментария факторно-расширенной векторной авторегрессии с изменяющимися во времени параметрами (TVP FAVAR). Гибкость данной модели заключается в возможности включения в модель неучтенных факторов, что позволяет добиваться более точных прогнозов по сравнению с SVAR. Также возможно проведение аналогичного представленному анализа с использованием векторной модели корректировки ошибок (VECM), поскольку между рядами были обнаружены общие детерминированные тренды.

\section{Библиографический список}

1. Breitung J., Bruggemann R., Lutkepohl H. Structural Vector Autoregressive Modeling and Impulse Responses. In Applied Time Series Econometrics / ed. by H. Lutkepohl, M. Kratzig. Cambridge: Cambridge University Press, 2004.

2. Gambetti L., Musso A. Loan supply shocks and the business cycle // Journal of Applied Econometrics. 2017, Vol. 32(4), pp. 764-782.

3. Kilian L., Lütkepohl H. Structural vector autoregressive analysis. Cambridge University Press, 2017.

4. Kilian L., Park C. The Impact of Oil Price Shocks on the U. S. Stock Market.// International Economic Review, 2009, Vol. 50(4), pp. 1267-1287.

5. King R.G., Plosser C. I., Stock J.H., Watson M. W. 1991. Stochastic Trends and Economic Fluctuations // American Economic Review, American Economic Association, 1991, Vol. 81(4), pp. 819-840.

6. Pfaff B. VAR, SVAR and SVEC Models: Implementation Within R Package vars // Journal of Statistical Software, 2008, vol. 27, pp. 1-32.

7. Sims C.A. Macroeconomics and reality // Econometrica, 1980, Vol. 48(1), pp. 1-48. 